Revue de droit comparé du travail et de la sécurité sociale

$3 \mid 2019$

Les migrations internationales de travail

\title{
La conciliation des vies familiale et professionnelle
}

\section{Vera Stangova}

\section{OpenEdition}

Journals

Édition électronique

URL : https://journals.openedition.org/rdctss/1529

DOI : $10.4000 /$ rdctss. 1529

ISSN : 2262-9815

Éditeur

Centre de droit comparé du travail et de la sécurité sociale

Édition imprimée

Date de publication : 1 novembre 2019

Pagination : 220-223

ISSN : 2117-4350

\section{Référence électronique}

Vera Stangova, «La conciliation des vies familiale et professionnelle», Revue de droit comparé du travail et de la sécurité sociale [En ligne], 3 | 2019, mis en ligne le 01 novembre 2021, consulté le 13 novembre 2021. URL : http://journals.openedition.org/rdctss/1529; DOI : https://doi.org/10.4000/rdctss.1529

\section{(c) $(9) \odot$}

Revue de droit comparé du travail et de la sécurité sociale est mise à disposition selon les termes de la Licence Creative Commons Attribution - Pas d'Utilisation Commerciale - Pas de Modification 4.0 International. 


\section{VERA STANGOVA}

Université Charles à Prague, Faculté de droit

\section{LA CONCILIATION DES VIES FAMILIALE ET PROFESSIONNELLE}

La conciliation vie familiale / professionnelle est au centre de l'intérêt des spécialistes du droit du travail et de la sécurité sociale, mais aussi des politiciens et surtout des salariés qui élèvent des enfants. Si les mesures prises concernent tous les salariés, c'est en pratique davantage le cas des femmes dont la participation à l'éducation des enfants est toujours plus importante que celle des hommes ${ }^{1}$.

Concilier la vie professionnelle et familiale présente de nombreuses difficultés, notamment pour les parents d'enfants en âge préscolaire. Pour résoudre cette situation souvent complexe, des solutions peuvent être offertes grâce aux formes d'emploi flexible ou atypique. À cet égard, le marché du travail en République tchèque accuse toujours un retard par rapport aux pays d'Europe occidentale.

Concernant la conciliation entre vie professionnelle et vie familiale, il existe un lien étroit entre le droit du travail et le droit de la sécurité sociale. Le Code du travail règlemente les obstacles qui peuvent survenir dans la vie du salarié (congé maternité, congé parental, etc.) et qui n'entraînent pas d'indemnisation du salaire perdu. En revanche, il revient au droit de la sécurité sociale d'assurer la sécurité matérielle du salarié lorsque surviennent ces événements empêchant de travailler².

Quelles sont les formes les plus connues d'emploi flexible? Une des formes à laquelle les femmes recourent souvent est le travail à temps réduit, bien qu'il ne soit pas réglementé par le droit du travail. II s'agit d'un travail d'une durée plus courte que la durée légale hebdomadaire du travail (40 heures par semaine en vertu du $\S 79$ alinéa 1 du Code du travail). Selon la disposition du § 241 alinéa 2 du même Code, les salariées enceintes, les travailleurs - hommes ou femmes - s'occupant d'un enfant de moins de 15 ans, et les autres travailleurs y mentionnés bénéficient d'un droit à la réduction de la durée de travail ou à tout autre aménagement convenable de la durée légale du travail hebdomadaire. Ce droit peut être accordé suite à leur demande préalable.

1 V. ŠTANGOVÁ, «Work-Life Balance in the Czech Republic », in J. Pichrt, K. Koldinská (dir.), Labour Law and Social Protection in a Globalized World, Changing Realities in Selected Areas of Law and Policy, Prague, Wolters Kluwer, 2018, p. 323.

2 V. ŠTANGOVÁ, "The Relationship between Social Security Law and Labour Law», The Lawyer Quarterly, Prague, 2017, p. 255. 


\section{I - HOME OFFICE, HOME WORKING}

Actuellement, le Home working n'est pas réglementé par le Code du travail. Les dispositions du § 317 précisent seulement que le travail peut aussi être exécuté en dehors des locaux de l'employeur. En pratique, cette modalité d'exécution du travail suscite de grandes attentes, pour les employeurs comme pour les travailleurs. Cette forme de travail peut être très avantageuse pour les travailleurs, mais elle peut également présenter certains risques. Selon les situations individuelles, il convient de prendre en considération les circonstances dans lesquelles il est possible de permettre au travailleur d'exécuter son travail à domicile avec la possibilité d'un aménagement flexible du temps de travail, ou au contraire, celles dans lesquelles sa présence sur le lieu du travail est indispensable.

\section{II - JOB SHARING (LE TRAVAIL PARTAGÉ)}

Une meilleure conciliation des vies professionnelle et familiale des salariés pourrait être rendue possible grâce à une nouvelle disposition juridique : le job sharing. Cette disposition est prévue dans le projet de modification du Code du travail dont l'entrée en vigueur initialement prévue le $1^{\text {er }}$ juillet 2019 a été retardée. La mesure proposée doit permettre qu'au moins deux travailleurs puissent, à leur initiative et suite à une convention préalable avec leur employeur, aménager leur temps de travail en collaboration et selon leurs besoins personnels respectifs, ce qui aboutirait à la création d'un poste de travail considéré comme "partagé ». La conclusion d'une convention individuelle entre l'employeur et chacun des travailleurs concernés reste une condition sine qua non de ce dispositif. Cette convention doit prévoir les modalités de l'aménagement du temps de travail et déterminer les intervalles de temps durant lesquels les travailleurs pourront organiser leurs horaires de travail, de manière à ce que l'activité requise par l'employeur soit assurée en continu.

\section{III - CONGÉ MATERNITÉ ET CONGÉ PARENTAL}

Ces dernières années, il a été constaté - notamment par les médias tchèques - que les durées du congé maternité (28 semaines ou 37 en cas de naissance multiple) et du congé parental (jusqu'aux 3 ans de l'enfant) figurent parmi les principales difficultés des femmes lors de la reprise du travail, et constituent un frein majeur à leur carrière professionnelle. Il faut toutefois signaler qu'il n'est pas obligatoire pour la mère de prendre l'intégralité du congé de maternité, mais seulement de respecter un minimum de 14 semaines, dont au moins 6 semaines après l'accouchement (selon le Code du travail). Bénéficier du reste du congé maternité est donc un droit, non un devoir. Dans ce contexte, il convient de souligner que, dans l'esprit des non juristes, les notions de congé maternité et de congé parental sont souvent confondues. Ainsi, ne fait-on pas souvent la distinction entre l'indemnité accordée lors du congé maternité (prestation du régime d'assurance-maladie) et la prestation accordée lors du congé parental (allocation du système d'aide sociale de l'État). En outre, ces différents dispositifs sont étonnamment désignés par l'appellation « maternité ». Cette confusion terminologique crée dans la pratique de nombreuses difficultés.

En vertu du $\S 196$ du Code du travail, l'employeur est obligé d'accorder, suite à la demande préalable, un congé parental au (à la) salarié(e) pour lui permettre de mieux soigner ses enfants. La mère peut bénéficier du congé parental dès la fin du congé de maternité, le père à partir de la naissance de l'enfant, et cela jusqu'aux trois ans de l'enfant. L'un des parents perçoit l'allocation parentale pour l'enfant le plus jeune dans la 
famille, à hauteur de 220000 couronnes tchèques (8500€) ou de 330000 (12750€) en cas de naissance multiple. A compter du $1^{\text {er }}$ janvier 2020, cette allocation sera portée à respectivement 300000 couronnes tchèques $(11600 €)$ et 450000 couronnes tchèques (17400€). Par ailleurs, l'allocation parentale pourra être octroyée au maximum jusqu'aux 4 ans de l'enfant.

En outre, le parent peut percevoir l'allocation parentale même s'il exerce une activité rémunérée, et cela sans tenir compte du niveau de revenu. En revanche, il doit assurer des soins réguliers, personnels et quotidiens au plus jeune enfant de la famille pour prétendre à cette prestation. Si l'enfant fréquente la crèche, l'école maternelle ou un établissement d'accueil préscolaire pour les enfants de moins de 2 ans, la prestation ne sera octroyée que si la présence de l'enfant dans ces établissements ne dépasse pas une certaine durée (loi n¹17/1995). La présence des enfants âgés de plus de 2 ans dans les établissements préscolaires n'est pas prise en considération.

Dès lors, l'organisation de cours et formations, destinés à la requalification des demandeurs d'emploi ou des parents en congé parental, soutenue par le Comité consultatif pour l'égalité des chances entre les femmes et les hommes, est d'une importance cruciale. Ces formations ont pour but de maintenir leur niveau de qualification et de faciliter ainsi leur retour sur le marché du travail. De même, il apparaît indispensable d'encourager les employeurs au maintien de liens et contacts avec leurs salariés en congé parental. Par ailleurs, le Comité pour la conciliation des vies personnelle, privée et familiale, qui opère dans le cadre de la Section pour l'égalité des chances entre les femmes et les hommes auprès du Bureau du Gouvernement de la République Tchèque, joue aussi un rôle important. Chargé d'analyser le contexte national dans le domaine de la conciliation des vies professionnelle et familiale, ce Comité propose des mesures adaptées.

\section{IV - LES ÉTABLISSEMENTS POUR LES ENFANTS D'ÂGE PRÉSCOLAIRE}

L'une des plus importantes barrières au retour des femmes au travail, au terme du congé maternité ou du congé parental, résulte de l'insuffisance des établissements préscolaires en termes de capacité et d'accessibilité. En effet, les capacités des établissements qui accueillent les enfants jusqu'à 3 ans ont considérablement diminué au début des années 90. À cette époque-là, il s'est répandu dans l'opinion que les soins apportés dans la famille étaient préférables pour les enfants en bas âge, notamment parce qu'il s'agit là d'une période où se forme le caractère de l'enfant et où les soins sont les plus exigeants. Pour cette raison, de nombreuses crèches et écoles maternelles ont été fermées. Cette tendance a perduré et au 31 décembre 2013, les crèches - en tant qu'établissements de santé publique - ont été soustraites de l'ordre juridique sans aucune compensation. Actuellement, il est de nouveau question de modifier la conception des soins apportés aux enfants jusqu'à l'âge de 3 ans. Certaines crèches subsistent encore et leur réintroduction généralisée est envisagée.

Au sein de I'Union européenne, une grande attention est portée aux questions relatives à la conciliation des vies professionnelle et familiale. On peut citer la Directive du Parlement européen et du Conseil de l'UE n² 2019/1158 du 20 juin 2019 concernant l'équilibre entre vie professionnelle et vie privée des parents et des aidants, qui abroge la Directive $n^{\circ}$ 2010/18 /UE du Conseil. A l'issue d'un délai de transposition triennal, il sera nécessaire d'adapter la législation nationale en fonction des modifications apportées par cette Directive: par exemple, l'insertion du travail à distance parmi les modalités de la 


\section{RÉPUBLIQUE TCHÈQUE}

relation de travail pouvant être demandées par les travailleurs/salariés qui s'occupent d'un enfant de moins de 15 ans, les travailleuses enceintes ou les salariés ayant une personne à charge. Dans ces cas, l'employeur devra donner suite à leur demande, à moins que cela ne cause de graves difficultés opérationnelles (§ 241 alinéa 2 du Code du travail). En outre, le congé parental devra être épuisé avant les 8 ans de l'enfant. La Directive réglemente aussi le droit des pères à prendre un court congé d'au moins 10 jours ouvrables pour la naissance de l'enfant.

Enfin, un énième problème se présente avec la différence entre la rémunération des hommes et celle des femmes. Ces dernières années, cette différence salariale se portait à plus de $20 \%$. De ce fait, la République tchèque occupe l'une des dernières places dans I'Union européenne. La rémunération inférieure des femmes se reflète également dans le niveau de vie des familles et, plus tard, dans le niveau des pensions de vieillesse octroyées aux femmes. 